\title{
NON-LINEARIZABILITY OF POLYNOMIALS AT IRRATIONALLY INDIFFERENT FIXED POINTS
}

\author{
YÛSUKe OKUYAMA
}

\begin{abstract}
In this paper, we consider the non-linearızability of polynomials with irrationally indifferent fixed points. Under the assumption that there exists a cubic polynomial which is linearizable at an irrationally indifferent fixed point with a non-Brjuno multiplier, we show that, for every degree more than two, one can construct a holomorphic family of possible maximal dimension consistıng of polynomials linearizable at the fixed point.
\end{abstract}

\section{Introduction}

Let $f$ be a germ of a holomorphic map at $z_{0} \in C$ with $f\left(z_{0}\right)=z_{0}$ and call $\lambda:=f^{\prime}\left(z_{0}\right)$ the multiplier of $f$ at $z_{0}$. We consider the linearization problem of $f$ at $z=z_{0}$, i.e. whether there exists a holomorphic local change of coordinate $z=h(w)$ with $h(0)=z_{0}$ and $h^{\prime}(0) \neq 0$ which conjugates $f$ to the linear map $w \mapsto \lambda w$. If such $h$ exists, the germ $f$ is said to be linearizable at $z_{0}$ and we call $h$ the (analytic) linearizing map of $f$ at $z_{0}$ or the solution of the linearization problem of $f$ at $z_{0}$.

If $\lambda=0$, Böttcher showed that $f(z)=z^{n}+a_{n+1} z^{n+1}+\cdots$ is always analytically conjugate to $w \mapsto w^{n}$. In the case $0<|\lambda|<1$ (resp. $1<|\lambda|$ ), Kœnigs showed that $f$ is always linearizable at $z_{0}$ and the fixed point $z_{0}$ is called attracting (resp. repelling). If $|\lambda|=1$ and $\lambda$ is a root of unity, $f$ is always non-linearizable at $z_{0}$ and $z_{0}$ is called parabolic (for the details, see [5]).

If $|\lambda|=1$ and $\lambda$ is not a root of unity, the fixed point $z_{0}$ of $f$ is said to be irrationally indifferent. In this case, some are linearizable at $z_{0}$, others nonlinearizable at $z_{0}$. For example, a rational or entire function $f$ which has an irrationally indifferent fixed point $z_{0}$ is linearizable there if and only if the fixed point $z_{0}$ belongs to the Fatou set of $f$ (cf. [5] and [8]).

From now on, we always assume that a real number $\alpha$ is irrational. Let

1991 Mathematics Subject Classification. Prımary 58F23, 30C62, 30D05, 39B12

Keywords and phrases. linearizability, irrationally indifferent fixed point, Brjuno number, cubiclike map.

Received April 23, 1998; revised August 13, 1998. 
$G_{z_{0}, \alpha}$ be the set of holomorphic germs at $z_{0}$ which have an irrationally indifferent fixed point $z_{0}$ with multiplier $\lambda=\exp (2 \pi i \alpha)$. If $\alpha$ is a Brjuno number, any germ $f \in G_{z_{0}, \alpha}$ is linearizable at $z_{0}([2])$. We define

$$
\mathscr{B}:=\{\alpha \in \boldsymbol{R}-\boldsymbol{Q} ; \alpha \text { is a Brjuno number }\} .
$$

The Brjuno numbers are defined in terms of the continued fractional expansion. Since we do not need the definition of them, we omit it. For the precise definition of them, see, for example, [5].

In [9] Yoccoz showed that the Brjuno condition is optimal. In other words, if $\alpha \notin \mathscr{B}$, we can find a germ $f \in G_{z_{0}, \alpha}$ which is non-linearizable at $z_{0}$, indeed, the quadratic polynomial $P(z)=e^{2 \pi l \alpha} z+z^{2}$ is non-linearizable at the origin. Thus it follows that a quadratic polynomial with an irrationally indifferent fixed point with multiplier $e^{2 \pi l \alpha}$ is linearizable there if and only if $\alpha$ is a Brjuno number, since it is affinely conjugate to $P(z)$. Can we extend this result for polynomials of degree more than two?

In this paper, we shall study the linearizability of polynomials of degree more than two at irrationally indifferent fixed points. family

In [7] Pérez-Marco showed that for $\lambda=\exp (2 \pi i \alpha)(\alpha \notin \mathscr{B})$ and $d>2$, the

$$
\mathscr{P}_{\lambda, d}:=\left\{P(z)=\lambda z+a_{2} z^{2}+\cdots+a_{d} z^{d} ;\left(a_{2}, \ldots, a_{d}\right) \in C^{d-1}\right\} \cong C^{d-1}
$$

contains an open dense subset whose elements are non-linearizable at the origin. It is not known whether this subset coincides with $\mathscr{P}_{\lambda, d}$.

The main theorem in this paper is the following.

MaIn Theorem. Fix $\lambda=e^{2 \pi i \alpha}(\alpha \notin \mathscr{B})$. Suppose there exists $A \in C$ such that the cubic polynomial $P_{\lambda, A}(z)=\lambda z+A z^{2}+z^{3}$ is linearizable at the origin, then for any $d \geq 3$, the family $\mathscr{P}_{\lambda, d}$ contains a holomorphic subfamily of complex dimension $d-2$ whose elements are linearizable at the origin.

Remark. Fix $\lambda=e^{2 \pi \iota \alpha}(\alpha \notin \mathscr{B})$. If $\mathscr{P}_{\lambda, d}$ has a nonlinear element $P_{d}$ linearizable at the origin, $(1 / c) P_{d}(c z)$ is also linearizable at the origin for any $c \in C^{*}$, so $\mathscr{P}_{\lambda, d}$ always contains a holomorphic subfamily of complex dimension one whose elements are linearizable at the origin.

By Pérez-Marco's result, if $\mathscr{P}_{\lambda, d}$ contains a holomorphic subfamily consisting of elements linearizable at the origin, the complex dimension of it is at most $d-2$. In this sense, Main Theorem says that for $d \geq 3, \mathscr{P}_{\lambda, d}$ contains a holomorphic subfamily of possible maximal dimension consisting of elements linearizable at the origin if it is impossible to extend Yoccoz's result on quadratic polynomials to the case of cubic polynomials. Thus we have the following.

Corollary 1.1. Fix $\lambda=e^{2 \pi i \alpha}(\alpha \notin \mathscr{B})$. The cubic polynomial $P_{\lambda, A}$ is nonlinearizable at the origin for any $A \in C$ if and only if the $\alpha$ satisfies the degeneration property: For some $d \geq 3, \mathscr{P}_{\lambda, d}$ does not contain a holomorphic subfamily of complex dimension $d-2$ whose elements are linearizable at the origin. 
COROLlaRY 1.2. If every non-Brjuno number has the degeneration property, it holds that a cubic polynomial with an irrationally indifferent fixed point with multiplier $e^{2 \pi i \alpha}$ is linearizable there if and only if $\alpha$ is a Brjuno number.

Remark. Fix $\lambda=e^{2 \pi \imath \alpha}(\alpha \notin \mathscr{B})$ and $d>3$. If $\mathscr{P}_{\lambda, d}$ has an element $P_{d}$ linearizable at the origin, by computing the dimension of Teichmüller space of the polynomial $P_{d}$ (cf. McMullen-Sullivan [4]), it may be possible to show a result similar to the above. But in this paper, we explicitly construct a holomorphic subfamily in the proof of Main Theorem.

In the rest of this paper, we shall prove Main Theorem. We fix $\lambda=e^{2 \pi l \alpha}$. In Section 2, we shall show that for a univalent function $f$ on $\boldsymbol{D}$ which has a fixed point $z=0$ with multiplier $\lambda$, the function $f_{a, A, b}(z):=a^{-1} f(a z)+A b z^{2}+b^{2} z^{3}$ can be regarded as a cubic-like map under a suitable condition. In Section 3, we shall show that the cubic-like map is quasiconformally conjugate to a cubic polynomial which has a fixed point at the origin with multiplier $\lambda$. In Section 4, we shall complete the proof of Main Theorem.

\section{Cubic perturbation of univalent maps}

We set

$$
\begin{aligned}
S:= & \{f ; \text { holomorphic and univalent functions on } \boldsymbol{D}, \\
& \left.\quad f(0)=0, \text { and }\left|f^{\prime}(0)\right|=1\right\} \\
S_{\lambda}:=\left\{f \in S ; f^{\prime}(0)=\lambda\right\} \text { and } & \\
\boldsymbol{D}_{r}:= & \{z ;|z|<r\} \text { for } r>0 .
\end{aligned}
$$

Let $\lambda=e^{2 \pi i \alpha}$ for $\alpha \in \boldsymbol{R}-\boldsymbol{Q}$, and let $f$ be an element of $S_{\lambda}$. We set, for $a \in \overline{\mathbf{D}}-\{0\}, A \in \boldsymbol{C}$ and $b \in \boldsymbol{C}$,

$$
f_{a, A, b}(z):=a^{-1} f(a z)+A b z^{2}+b^{2} z^{3} .
$$

A triplet $(\tilde{U}, U, f)$ is called a cubic-like map if $\tilde{U}$ and $U$ are simply connected proper subdomains of $C$, and $\tilde{U}$ is relatively compact in $U$, and $f: \tilde{U} \rightarrow U$ is a proper holomorphic map of degree 3.

Lemma 2.1. For $A \in C$ and $b \in C$, we define

$$
\begin{aligned}
R_{A, b} & :=\frac{10}{9}|A||b|+\frac{15}{2} \\
B_{A, b} & :=27 R_{A, b}+3|A||b|+\frac{81}{4}=33|A||b|+\frac{891}{4} \\
W & :=D_{R_{A, b}}=\left\{z ;|z|<R_{A, b}\right\} \quad \text { and } \\
\tilde{W}: & =D_{1 / 3} \cap f_{a, A, b}^{-1}(W) .
\end{aligned}
$$


For $f \in S, a \in \overline{\mathbf{D}}-\{0\}, A \in C$ and $|b|^{2}>B_{A, b}$, the triplet $\left(\tilde{W}, W, f_{a, A, b}\right)$ is a cubic-like map.

Proof. It is sufficient to prove this in the case $a=1$. Since $f \in S$, it follows that $|z| /(1+|z|)^{2} \leq|f(z)| \leq|z| /(1-|z|)^{2}$ for $z \in D$. In particular, if $|z|=1 / 3$, we have $3 / 16 \leq|f(z)| \leq 3 / 4$ and it follows that

$$
\left|f_{1, A, b}(z)\right| \geq\left|b^{2} z^{3}\right|-\left|A b z^{2}+f(z)\right| \geq \frac{|b|^{2}}{27}-\frac{|A||b|}{9}-\frac{3}{4}>R_{A, b} .
$$

Thus $f_{1, A, b}\left(\boldsymbol{D}_{1 / 3}\right)$ contains the disk $\bar{W}$, so $f_{1, A, b}: \tilde{W} \rightarrow W$ is proper and $\tilde{W}$ is simply connected by the maximum modulus principle. And for $|z|=1 / 3$ and $z_{1} \in W$, it follows from (1) that

$$
\begin{gathered}
\left|b^{2} z^{3}-z_{1}\right|>\left|b^{2} z^{3}\right|-R_{A, b}>\left|A b z^{2}+f(z)\right| \text { and } \\
\sqrt[3]{\left|\frac{z_{1}}{b^{2}}\right|}<\sqrt[3]{\frac{R_{A, b}}{|b|^{2}}}<\sqrt[3]{\frac{R_{A, b}}{27 R_{A, b}}}=\frac{1}{3}
\end{gathered}
$$

since $|b|^{2}>B_{A, b}>27 R_{A, b}$ by definition. Thus by the theorem of Rouché, $f_{1, A, b}$ : $\tilde{W} \rightarrow W$ is a proper map of degree 3 .

If $\tilde{W}$ is not connected, then the number of connected components of $\tilde{W}$ is three or two. First, if it is three, the connected component of $\tilde{W}$ containing the origin is conformally mapped to $W$ by $f_{1, A, b}$. However this contradicts the Schwarz lemma because $|\lambda|=1$. Second, if it is two, two cases occur. If $f_{1, A, b}$ conformally maps the connected component of $\tilde{W}$ containing the origin onto $W$, we can derive a contradiction by the same argument as above. Otherwise $f_{1, A, b}$ conformally maps the other component $W^{\prime}$ of $\tilde{W}$ onto $W$. So there exists the only one point $z_{0} \in W^{\prime}$ such that $f_{1, A, b}\left(z_{0}\right)=0$. We define $\phi:=\left(f_{1, A, b} \mid W^{\prime}\right)^{-1}$, and $\psi(z):=\phi\left(R_{A, b} z\right)$. Then $\psi$ conformally maps $\boldsymbol{D}$ onto $W^{\prime}$, and $\psi(0)=z_{0}$ (see Figure 1).

By the Koebe one-quarter theorem, it follows that $W^{\prime}$ contains the open disk of which the radius is $(1 / 4)\left|\psi^{\prime}(0)\right|=(1 / 4) R_{A, b}\left|\phi^{\prime}(0)\right|$. Since $D_{1 / 3} \supset W^{\prime}$ and $W^{\prime} \not \nexists 0$, we have $(1 / 4) R_{A, b}\left|\phi^{\prime}(0)\right|<1 / 6$. Hence

$$
\frac{1}{\left|\phi^{\prime}(0)\right|}>\frac{3 R_{A, b}}{2} \text {. }
$$

On the other hand, we have

$$
\frac{1}{\left|\phi^{\prime}(0)\right|}=\left|f_{1, A, b}^{\prime}\left(z_{0}\right)\right| \leq\left|f^{\prime}\left(z_{0}\right)\right|+2|A||b|\left|z_{0}\right|+3|b|^{2}\left|z_{0}\right|^{2}
$$

and by the Koebe distortion theorem, $\left|f^{\prime}\left(z_{0}\right)\right|<9 / 2$ for $\left|z_{0}\right|<1 / 3$. Since $f_{1, A, b}\left(z_{0}\right)=f\left(z_{0}\right)+A b z_{0}^{2}+b^{2} z_{0}^{3}=0$ and $\left|f\left(z_{0}\right)\right| \leq\left|z_{0}\right| /\left(1-\left|z_{0}\right|\right)^{2}$, we have $3|b|^{2}\left|z_{0}\right|^{2}+$ 


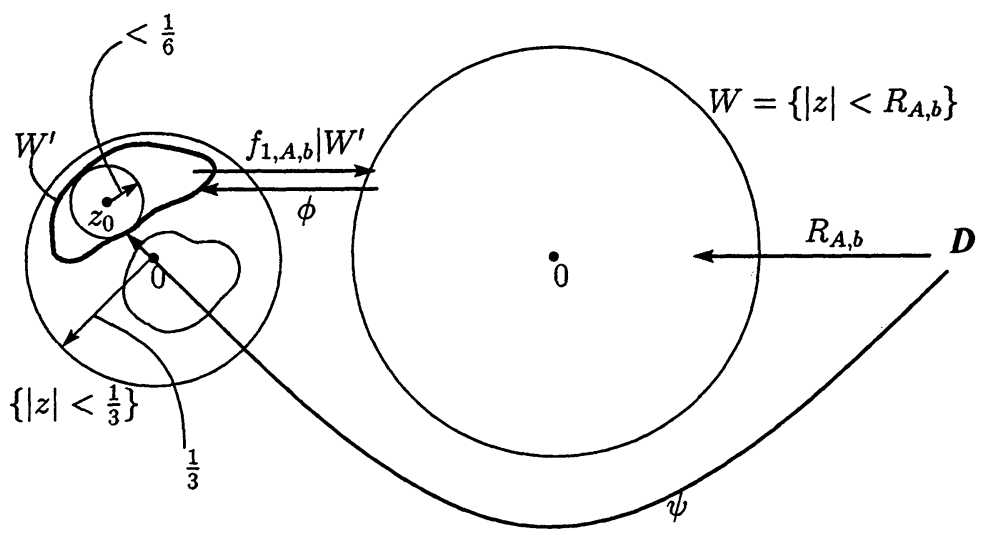

Figure 1

$2|A||b|\left|z_{0}\right|<(27 / 4)+(5 / 3)|A||b|$ for $\left|z_{0}\right|<1 / 3$. Hence

$$
\frac{1}{\left|\phi^{\prime}(0)\right|}<\frac{9}{2}+\frac{27}{4}+\frac{5}{3}|A||b|=\frac{45}{4}+\frac{5}{3}|A||b|=\frac{3}{2} R_{A, b} .
$$

This is a contradiction. So $\tilde{W}$ is connected, and the proof is completed.

\section{Straightening of the cubic-like mapping}

We define $N(M):=\left(33 M+\sqrt{1089 M^{2}+891}\right) / 2$ for $M>0$. Then $|b|^{2}>$ $B_{A, b}$ if and only if $|b|>N(|A|)$.

Let $M$ be an arbitrary positive number and take a smooth function $\eta: \boldsymbol{R} \rightarrow[0,1]$ which is identically 1 on $(-\infty, 1 / 3]$ and 0 on $\left[R_{A, b},+\infty\right)$. And we define the round annulus $\boldsymbol{A}(M):=\{b ; N(M)<|b|<N(M)+1\}$. There exists a constant $R(M)>0$ which depends only on $M$ such that $R_{A, b}<R(M)$ for any $A \in \boldsymbol{D}_{M}$ and $b \in \boldsymbol{A}(M)$.

For $f \in S_{\lambda}, a \in \overline{D_{1 / R(M)}}-\{0\}, A \in D_{M}$ and $b \in A(M)$, we define

$$
\tilde{f}_{a, A, b}(z):=\eta(|z|) f_{a, A, b}(z)+(1-\eta(|z|))\left(\lambda z+A b z^{2}+b^{2} z^{3}\right) .
$$

Then $\tilde{f}_{a, A, b}: C \rightarrow C$ is in $C^{\infty}$.

Lemma 3.1. If $a \rightarrow 0$, then $\tilde{f}_{a, A, b}(z)$ converges to $\lambda z+A b z^{2}+b^{2} z^{3}$ in $C^{\infty}$ topology on $C$, and this convergence is uniform in $f \in S_{\lambda}, A \in D_{M}$ and $b \in A(M)$.

Proof. On $\boldsymbol{C}-\boldsymbol{D}_{R_{A, b}}, \tilde{f}_{a, A, b}(z) \equiv \lambda z+A b z^{2}+b^{2} z^{3}$. If we write $f(z)=\lambda z+$ $\sum_{n=2}^{\infty} a_{n} z^{n}$ then $\left|a_{n}\right| \leq n$. Noting that $R_{A, b}<R(M)$, we see that on $D_{R_{A, b}}$, 


$$
\begin{aligned}
\left|f_{a, A, b}-\left(\lambda z+A b z^{2}+b^{2} z^{3}\right)\right| & \leq \sum_{n=2}^{\infty}\left|a_{n}\right||a|^{n-1}|z|^{n} \\
& \leq|z| \sum_{n=2}^{\infty} n|a z|^{n-1} \leq R(M) \sum_{n=2}^{\infty} n|a R(M)|^{n-1} \\
& <R(M) \frac{|a| R(M)(2-|a| R(M))}{(1-|a| R(M))^{2}}
\end{aligned}
$$

so $\lim _{a \rightarrow 0} f_{a, A, b}(z)=\lambda z+A b z^{2}+b^{2} z^{3}$ uniformly on $\boldsymbol{D}_{R_{A, b}}$ and in $f \in S_{\lambda}$, $A \in \boldsymbol{D}_{M}$ and $b \in \boldsymbol{A}(M)$. Since $\tilde{f}_{a, A, b}(z)-\left(\lambda z+A b z^{2}+b^{2} z^{3}\right)=\eta(z)\left\{f_{a, A, b}(z)-\right.$ $\left.\left(\lambda z+A b z^{2}+b^{2} z^{3}\right)\right\}$, it follows that $\lim _{a \rightarrow 0} \tilde{f}_{a, A, b}(z)=\lambda z+A b z^{2}+b^{2} z^{3}$ uniformly on $\boldsymbol{C}$ and in $f \in S_{\lambda}, A \in \boldsymbol{D}_{M}$ and $b \in \boldsymbol{A}(M)$. Similarly, we can see the convergence of the derivatives of $\tilde{f}_{a, A, b}$.

LeMma 3.2. Both of two finite critical points of $\tilde{f}_{a, A, b}(z)$ belong to $\{z ;|z|<1 / 3\}$ if $|a|$ is sufficiently small.

Proof. From Lemma 3.1, it is sufficient to prove that two critical points of $z \mapsto \lambda z+A b z^{2}+b^{2} z^{3}$ is included in $\{z ;|z|<1 / 3\}$. Note that $\left(\lambda z+A b z^{2}+b^{2} z^{3}\right)^{\prime}=$ $\lambda+2 A b z+3 b^{2} z^{2}$. Since $|b|>N(M)$ i.e. $|b|^{2}>B_{A, b}$, we can see on $|z|=1 / 3$,

$$
\left|3 b^{2} z^{2}\right|-|\lambda+2 A b z| \geq \frac{B_{A, b}}{3}-1-\frac{2}{3}|A||b|>0 .
$$

By the theorem of Rouché, both of critical points of $\lambda z+A b z^{2}+b^{2} z^{3}$ are in $D_{1 / 3}$.

We can conclude the following by the previous lemmas.

Lemma 3.3. There exist an $a_{0} \in(0,1 / R(M)]$ and a continuous function $k$ : $\left[0, a_{0}\right] \rightarrow[0,1)$ such that $k(0)=0$ and for any $f \in S_{\lambda}, A \in D_{M}, b \in A(M)$ and $a \in$ $\overline{\boldsymbol{D}}_{a_{0}}-\{0\}$, the map $\tilde{f}_{a, A, b}$ is a branched covering map of $\boldsymbol{C}$ of degree 3 and it satisfies

$$
\left|\frac{\bar{\partial} \tilde{f}_{a, A, b}(z)}{\partial \tilde{f}_{a, A, b}(z)}\right| \leq k(|a|) \quad\left(\frac{1}{3} \leq|z| \leq R_{A, b}\right) .
$$

Moreover, the Beltrami coefficient $\bar{\partial} \tilde{f}_{a, A, b}(z) / \partial \tilde{f}_{a, A, b}(z)$ holomorphically depends on $f \in S_{\lambda}, A \in \boldsymbol{D}_{M}, b \in \boldsymbol{A}(M)$ and $a \in \boldsymbol{D}_{a_{0}}-\{0\}$.

For an open set $U \subset C$, we identify a Beltrami coefficient on $U$ with a function $\mu \in L^{\infty}(U)$ such that $\|\mu\|_{\infty}<1$. And for a $C^{1}$-function $f: U \rightarrow V$ and a Beltrami coefficient $\mu$ on $V$, we define the pullback $f^{*} \mu$ of $\mu$ on $U$ by

$$
\left(f^{*} \mu\right)(z)=\frac{\overline{\partial f(z)} \mu(f(z))+\bar{\partial} f(z)}{\overline{\bar{\partial} f(z)} \mu(f(z))+\partial f(z)} .
$$


For $f \in S_{\lambda}, A \in D_{M}, b \in A(M)$ and $a \in D_{a_{0}}-\{0\}$, there exists a unique Beltrami coefficient $\mu=\mu_{f, a, A, b}$ on $C$ which is invariant under the pullback by $\tilde{f}_{a, A, b}$ and agrees with $\bar{\partial} \tilde{f}_{a, A, b} / \partial \tilde{f}_{a, A, b}$ on $1 / 3 \leq|z| \leq R_{A, b}$ and is 0 on $(\boldsymbol{C}-\boldsymbol{W}) \cup$ $\bigcap_{n \geq 0} f_{a, A, b}^{-n}(\tilde{W})$. Since $\operatorname{supp} \mu \subset W$ and $\|\mu\|_{\infty} \leq k(a)<1$, by the Ahlfors-Bers theorem [1], there exists a unique quasiconformal homeomorphism $\phi=\phi_{f, a, A, b}$ of $C$ onto itself which satisfies the following

(i) for a.e. $z \in C, \bar{\partial} \phi(z)=\mu(z) \partial \phi(z)$,

(ii) $\phi(0)=0$ and

(iii) $\phi(z)-z$ is bounded on $C$.

LeMma 3.4 (cf. [3]). There exists an $A^{\prime} \in C$ such that $\phi \circ \tilde{f}_{a, A, b} \circ \phi^{-1}(z)=$ $\lambda z+A^{\prime} z^{2}+b^{2} z^{3}$, where $A^{\prime} \in C$ holomorphically depends on $f \in S_{\lambda}, A \in D_{M}, b \in$ $\boldsymbol{A}(M)$ and $a \in \boldsymbol{D}_{a_{0}}-\{0\}$.

Proof. Since $\mu\left(\phi \circ f_{a, A, b}\right)=\mu(\phi)$, it follows that $\phi \circ \tilde{f}_{a, A, b} \circ \phi^{-1}: C \rightarrow \boldsymbol{C}$ is holomorphic and fixes the origin. So it is a branched covering map of $C$ of degree 3 fixing the origin. Thus we can write

$$
\phi \circ \tilde{f}_{a, A, b} \circ \phi^{-1}(z)=\lambda^{\prime} z+A^{\prime} z^{2}+b^{\prime} z^{3} \quad\left(\lambda^{\prime}, A^{\prime}, b^{\prime} \in C\right) .
$$

By the theorem of Naishul [6], the multiplier of the fixed point of a holomorphic map is topologically invariant when its modulus is 1 . So we have $\lambda^{\prime}=\lambda$. Next, we show $b^{\prime}=b^{2}$. According to (iii), we have

$$
\phi_{f, a, A, b}(z)=z+c+(\text { lower terms })
$$

on a neighborhood of the point at infinity. When $|z|$ is sufficiently large, $\tilde{f}_{a, A, b}(z)=\lambda z+A b z^{2}+b^{2} z^{3}$ by definition, and we note that $\phi\left(\tilde{f}_{a, A, b}(z)\right)=\lambda \phi(z)+$ $A^{\prime}(\phi(z))^{2}+b^{\prime}(\phi(z))^{3}$. Therefore it follows that

$$
\begin{aligned}
\phi(\lambda z & \left.+A b z^{2}+b^{2} z^{3}\right)-\left(\lambda z+A b z^{2}+b^{2} z^{3}\right) \\
& =\left(b^{\prime}-b^{2}\right) z^{3}+\left\{\left(A^{\prime}-A b\right)+3 b^{\prime} c\right\} z^{2}+\text { (lower terms). }
\end{aligned}
$$

Since this quantity remains bounded as $|z| \rightarrow+\infty$, it is necessary that $b^{\prime}-b^{2}=0$ and $A^{\prime}-A b+3 b^{\prime} c=0$. Thus it follows that $b^{\prime}=b^{2}$ and $A^{\prime}=A b-3 b^{2} c$.

Lemma 3.5. $c=c(f, a, A, b)$ in (2) holomorphically depends on $f \in S_{\lambda}, A \in$ $\boldsymbol{D}_{M}, b \in \boldsymbol{A}(M)$ and $a \in \boldsymbol{D}_{a_{0}}-\{0\}$. And $c \rightarrow 0$ uniformly in $f \in S_{\lambda}, A \in \boldsymbol{D}_{M}$ and $b \in \boldsymbol{A}(M)$ as $a \rightarrow 0$.

Proof. The former part follows from the Ahlfors-Bers theorem. Noting that $\|\mu\|_{\infty} \leq k(|a|)$ and $\lim _{a \rightarrow 0} k(|a|)=0$ uniformly in $f \in S_{\lambda}, A \in D_{M}$ and $b \in$ $\boldsymbol{A}(\boldsymbol{M})$, we can see the latter part holds. 


\section{Proof of Main Theorem}

Let $\alpha \notin \mathscr{B}$ and $\lambda=e^{2 \pi i \alpha}$. For $A_{0} \in C$, we take $M=M_{0}:=2\left|A_{0}\right|+1$. By Lemma 3.5, for a fixed $0<\varepsilon<1 / 3$, there exists an $a_{1} \in\left(0, a_{0}\right]$ which is independent of $f \in S_{\lambda}, A \in D_{M_{0}}$ and $b \in A\left(M_{0}\right)$ such that

$$
3|b||c(f, a, A, b)|<\varepsilon \quad\left(0<|a|<a_{1}\right) .
$$

Then $\left|A_{0}\right|<M_{0}-2 \varepsilon$. We define a holomorphic map $F_{f, a, b}$ on $\boldsymbol{D}_{M_{0}}$ :

$$
A \mapsto A-3 b c(f, a, A, b) .
$$

By the theorem of Rouché, there exists $A_{1}=A_{1}(f, a, b) \in D_{M_{0}-\varepsilon}$ such that $F_{f, a, b}\left(A_{1}\right)=A_{0}$. From the implicit function theorem, $A_{1}=A_{1}(f, a, b)$ holomorphically depends on $f \in S_{\lambda}, b \in \boldsymbol{A}\left(M_{0}\right)$ and $a \in \boldsymbol{D}_{a_{1}}-\{0\}$. Thus we have the following.

THEOREM 4.1. Fix $\lambda=e^{2 \pi l \alpha}(\alpha \notin \mathscr{B})$. For $A_{0} \in C$, we set $M_{0}:=2\left|A_{0}\right|+1$. Then there exist an $a_{1} \in\left(0, a_{0}\right]$, a holomorphic function $A_{1}=A_{1}(f, a, b)$ with $\left|A_{1}\right|<M_{0}$ of $f \in S_{\lambda}, a \in \boldsymbol{D}_{a_{1}}-\{0\}, b \in \boldsymbol{A}\left(M_{0}\right)$, and also exists a quasiconformal homeomorphism $\phi=\phi_{f, a, A_{1}, b}$ of $\boldsymbol{C}$ onto itself such that

$$
\phi \circ \tilde{f}_{a, A_{1}, b} \circ \phi^{-1}(z)=\lambda z+A_{0} b z^{2}+b^{2} z^{3}=\frac{1}{b} P_{\lambda, A_{0}}(b z) .
$$

If $P_{\lambda, A_{0}}$ is linearizable at the origin, then $f_{a, A_{1}, b}(z)=a^{-1} f(a z)+A_{1} b z^{2}+b^{2} z^{3}$ is linearizable at the origin.

Proof. The former part has been proved in the above and the latter part follows from Lemma 3.4. Assume $P_{\lambda, A_{0}}$ is linearizable at the origin and let $D$ be the Siegel disk of $(1 / b) P_{\lambda, A_{0}}(b z)$. We have $f_{a, A_{1}, b}\left(\phi^{-1}(D)\right)=\phi^{-1}(D)$, so the fixed point $z=0$ of $f_{a, A_{1}, b}$ is contained in the Fatou set of $f_{a, A_{1}, b}$.

Proof of Main Theorem. Suppose $P_{\lambda, A_{0}}$ is linearizable at the origin. If $d=$ 3, Main Theorem is trivial. We consider, for $d>3$, the family

$$
\mathscr{U}_{d}:=\left\{P(z)=\lambda z+a_{2} z^{2}+\cdots+a_{d} z^{d} ; \sum_{n=2}^{d} n\left|a_{n}\right| \leq 1\right\} \subset S_{\lambda} .
$$

We fix $b \in \boldsymbol{A}\left(M_{0}\right)$ and $a \in \boldsymbol{D}_{a_{1}}-\{0\}$. If $f(z)=\lambda z+a_{2} z^{2}+a_{3} z^{3}+\cdots+a_{d} z^{d} \in$ $\mathscr{U}_{d}$ and $a_{d} \neq 0$, then we can see

$$
f_{a, A_{1}, b}(z)=\lambda z+\left(a_{2} a+A_{1} b\right) z^{2}+\left(a_{3} a^{2}+b^{2}\right) z^{3}+\sum_{n=4}^{d} a_{n} a^{n-1} z^{n}
$$

is linearizable at the origin. The map

$$
\left(a_{2}, a_{3}, \ldots, a_{d}\right) \mapsto\left(a_{2} a+A_{1}\left(a, b, a_{2}, a_{3}, \ldots, a_{d}\right) b, a_{3} a^{2}+b^{2}, a_{4} a^{3}, \ldots, a_{d} a^{d-1}\right)
$$

is holomorphic on $\left\{\left(a_{2}, a_{3}, \ldots, a_{d}\right) ; \sum_{n=2}^{d} n\left|a_{n}\right|<1\right.$ and $\left.a_{d} \neq 0\right\}$ which is an open 
subset of $C^{d-1}$. Note that the Jacobian of the map $\left(a_{3}, \ldots, a_{d}\right) \mapsto\left(a_{3} a^{2}+b^{2}\right.$, $\left.a_{4} a^{3}, \ldots, a_{d} a^{d-1}\right)$ is not zero. Therefore $\left\{f_{a, A_{1}, b} ; f \in \mathscr{U}_{d}\right\}$ is at least a $d-2$ dimensional holomorphic subfamily of $\mathscr{P}_{\lambda, d}$ and all of the elements are linearizable at the origin. Consequently we have completed the proof of Main Theorem in Section 1.

In Proof of Main Theorem, we do not use Pérez-Marco's result. Combining Pérez-Marco's result and the argument in Proof of Main Theorem, we have the following.

TheOREM 4.2. Fix $\lambda=e^{2 \pi i \alpha}(\alpha \notin \mathscr{B})$. The cubic polynomial $P_{\lambda, A_{0}}(z)=\lambda z+$ $A_{0} z^{2}+z^{3}$ is non-linearizable at the origin if the pair $\left(\alpha, A_{0}\right)$ satisfies the fulldimension property: For some $d>3$, the holomorphic map

$$
\begin{aligned}
& \left(a, b, a_{2}, \ldots, a_{d}\right) \mapsto \\
& \quad\left(a_{2} a+A_{1}\left(a, b, a_{2}, a_{3}, \ldots, a_{d}\right) b, a_{3} a^{2}+b^{2}, a_{4} a^{3}, \ldots, a_{d} a^{d-1}\right)
\end{aligned}
$$

has a maximal rank $d-1$.

Proof. Suppose that $P_{\lambda, A_{0}}$ is linearizable at the origin. Then the image of the map (3) is a $d-1$ dimensional holomorphic subfamily of $\mathscr{P}_{\lambda, d}$ consisting of elements linearizable at the origin. It contradicts Pérez-Marco's result.

COROllary 4.1. If any pair $\left(\alpha, A_{0}\right)\left(\alpha \notin \mathscr{B}\right.$ and $\left.A_{0} \in C\right)$ has the fulldimension property, it holds that a cubic polynomial with an irrationally indifferent fixed point with multiplier $e^{2 \pi i \beta}$ is linearizable there if and only if $\beta$ is a Brjuno number.

Acknowledgement. The author would like to express his gratitude to Prof. Masahiko Taniguchi and Prof. Toshiyuki Sugawa for many valuable discussions and advices.

\section{REFERENCES}

[1] L. V Ahlfors AND L. Bers, Riemann's mapping theorem for variable metrics, Ann. of Math., 72 (1960), 385-404.

[2] A. D. Bruuno, Analytical form of differential equations, Trans. Moscow Math. Soc., 25 (1971), 199-239.

[3] A. Douady and J. H. Hubbard, On the dynamics of polynomial-like mappings, Ann. Sci., Ecole Norm. Sup. (4), 18 (1985), 287-343.

[4] C. MCMullen AND D. Sullivan, Quasiconformal homeomorphisms and dynamics III: The Teichmüller space of a holomorphıc dynamıcal system, preprint.

[5] J. Milnor, Dynamics in one complex variable, preprint.

[6] V I. NaISHUL, Topological invariants of analytic and area preserving mappings and their application to analytic differential equations in $\boldsymbol{C}^{2}$ and $\boldsymbol{C P}^{2}$, Trans. Moscow Math. Soc., 42 (1983), 239-250. 
[ 7 ] R. Ṕ́rez-Marco, Sur les dynamique holomorphes non linéarıables et une conjecture de V. I. Arnold, Ann. Sci. École Norm Sup. (4), 26 (1993), 565-644.

[ 8 ] R. Pérez-Marco, Fixed points and circle maps, Acta Math., 179 (1997), 243-294.

[9] J. C. Yoccoz, Thèorém de Siegel, nombres de Bruno et polynômes quadratıques, Astérisque, 231 (1996), 3-88.

Department of Mathematics

Graduate SchoOl of SCIENCE

KYOTO UNIVERSITY

Куото 606-8502

JAPAN

E-mail: okuyama@kusm.kyoto-u.ac.jp 